\title{
10. OXYGEN AND CARBON ISOTOPIC RECORDS FROM THE OOZES OF SITES 752, 754, 756, AND 757, EASTERN INDIAN OCEAN
}

\author{
David K. Rea, ${ }^{2}$ K. C. Lohmann, ${ }^{2}$ Norman D. MacLeod, ${ }^{2,3}$ Martha A. House, ${ }^{2}$ Steven A. Hovan, ${ }^{2}$ and Gale D. Martin ${ }^{2}$
}

\begin{abstract}
Oxygen and carbon isotopic records have been developed for the Cenozoic carbonate oozes of Sites 752, 754, 756, and 757 based on the analysis of monospecific benthic foraminifers. The intent of this report is to provide a basic isotopic stratigraphy for use in other paleoceanographic studies. The oxygen isotope record displays the enrichments associated with cooling or ice volume buildup at the Eocene/Oligocene boundary, in the middle Miocene, and in the upper Pliocene. The carbon isotopic record contains the Chron 16 enrichment in the lower Miocene and the Chron 6 depletion in the uppermost Miocene.
\end{abstract}

\section{INTRODUCTION}

The upper sedimentary unit recovered in each of the Ocean Drilling Program (ODP) Leg 121 drill sites is a carbonate ooze. We have chosen the stratigraphically longer cores from Broken Ridge, Sites 752 and 754, and Sites 756 and 757 from the southern end of the Ninetyeast Ridge for an isotopic survey of these Cenozoic materials (Fig. 1, Table 1). The intent of our analyses is to provide a carbon and oxygen isotopic stratigraphy for these low sedimentation-rate cores so that any paleoceanographic observations derived from them can be placed into the global record of climatic and oceanographic changes. The general depositional records of these sites were described by Peirce, Weissel, et al. (1989); Rea et al. (1990) presented an overview of the paleoceanographic record of the eastern Indian Ocean interpreted from the sedimentary section of Broken Ridge.

\section{SEDIMENTS}

The plateau region of Broken Ridge is underlain by a foraminifer or foraminifer-bearing nannofossil ooze. This ooze reaches a maximum thickness of about $120 \mathrm{~m}$ at Site 754 , near the center of the platform, and thins to the north and south. A layer of sand and limestone pebbles denotes a lower Oligocene disconformity and divides the ooze into upper and lower portions. The upper ooze unit, from which most of our samples are derived, ranges in age from Pleistocene to middle Oligocene. The ooze below the Oligocene disconformity, of late Eocene age, was poorly recovered and we have only a few samples from that interval. The scientists of Deep Sea Drilling Project (DSDP) Leg 26, which also drilled on Broken Ridge, believed that those carbonate oozes, now at 1050 to $1100 \mathrm{~m}$ water depth, showed evidence of winnowing (Davies, Luyendyk, et al., 1974). Our shipboard work tended to confirm that observation (Rea et al., 1990; House et al., this volume). The implication of important amounts of winnowing to this work is that in these slowly accumulating sediments there may be small hiatuses that are unrecog-

\footnotetext{
${ }^{1}$ Weissel, J., Peirce, J., Taylor, E., Alt, J., et al., 1991. Proc. ODP, Sci. Results, 121: College Station, TX (Ocean Drilling Program).

${ }^{2}$ Department of Geological Sciences, The University of Michigan, Ann Arbor, MI 48109-1063, U.S.A

${ }^{3}$ Current address: Department of Geological and Geophysical Sciences, Princeton University, Princeton, NJ 08540, U.S.A.
}

nized at the relatively widely spaced sampling intervals used by the several investigators.

At Site 756 on the southern end of Ninetyeast Ridge, $144.5 \mathrm{~m}$ of nannofossil ooze containing $5 \%$ to $20 \%$ foraminifers overlies about $6 \mathrm{~m}$ of foraminifer limestone, which rests on basalt. The foraminifer-bearing nannofossil ooze is Pleistocene to late Eocene in age. Trace amounts of volcanic ash occur throughout the ooze, with enhanced ash concentrations between about 50 and 63 $\mathrm{m}$ below seafloor (mbsf). At Site 757, $212 \mathrm{~m}$ of nannofossil ooze, becoming more micritic and chalky below about $169 \mathrm{mbsf}$, overlie basalt. The ooze at Site 757 ranges in age from Pleistocene to early Eocene.

The depths of the Broken Ridge drill sites have always been relatively shallow. The ridge was uplifted and exposed to erosion in the middle Eocene and probably was exposed again in the middle Oligocene (Peirce, Weissel, et al., 1989; Rea et al., 1990). Since then the ridge crest has subsided to its present depth of about $1100 \mathrm{~m}$. The depth of $1100 \mathrm{~m}$ approximates that of the boundary between the Surface Water and Antarctic Intermediate Water masses, near the bottom of the main thermocline. The significance of the subsidence history is that these sites have always been in the Surface Water Mass and so the benthic foraminifers record that isotopic history rather than the record of deep or bottom waters. Site 756 on Ninetyeast Ridge is at $1529 \mathrm{~m}$ and Site 757 is at $1652 \mathrm{~m}$, both within the Antarctic Intermediate Water Mass. The volcanic rocks beneath the sediments at Sites 756 and 757, of Eocene and late Paleocene age, respectively, show evidence for subaerial eruption and weathering (Peirce, Weissel, et al., 1989) suggesting that the materials at these sites also represent in large part a record of the Surface Water Mass.

In this discussion we will refer to the nannofossil stratigraphy as determined aboard the JOIDES Resolution and reported by Peirce, Weissel, et al. (1989). The zonations were referred to the mid-latitude biostratigraphic scheme of Bolli et al. (1985), which is slightly different than the time scale of Berggren et al. (1985), especially in the Miocene. For instance, the lower/middle Miocene boundary in the Bolli et al. (1985) time scale is at the CN4/CN5 boundary, $15.4 \mathrm{Ma}$; the same boundary in the Berggren et al. (1985) time scale is between nannofossil Zones CN3 and $\mathrm{CN} 4$, at 16.5 Ma. Persons interested in the details of the biostratigraphy should consult Peirce, Weissel, et al. (1989). Additional information on nannofossil zonations in the Miocene was provided by P. Resiwati (pers. comm., 1990). 


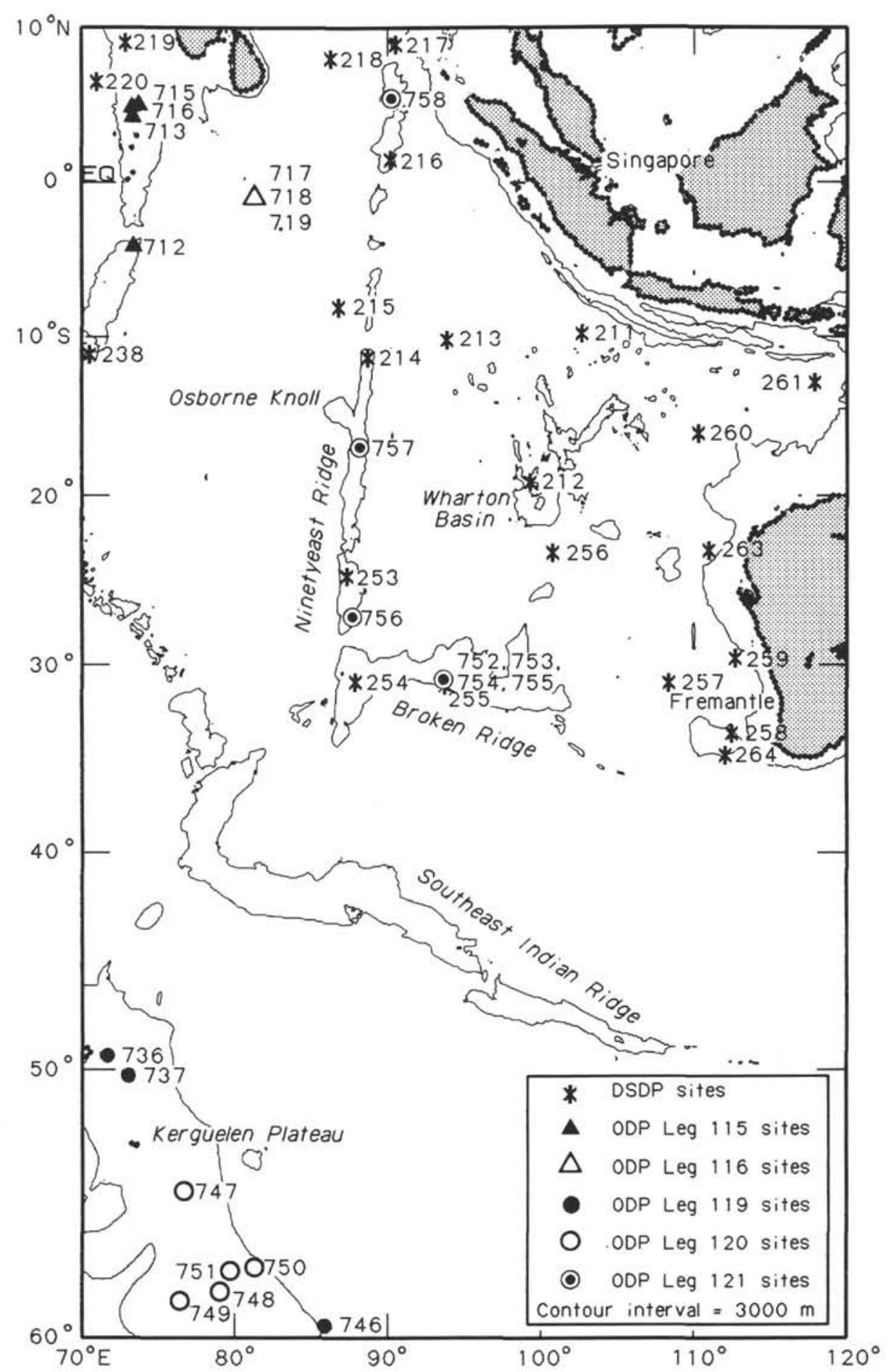

Figure 1. Index map of the eastern Indian Ocean showing the location of Leg 121 drill sites and other ODP/DSDP sites. 
Table 1. Location and water depth of Leg 121 drill sites studied.

\begin{tabular}{cccc}
\hline Hole & $\begin{array}{c}\text { Latitude } \\
(\mathrm{S})\end{array}$ & $\begin{array}{c}\text { Longitude } \\
(\mathrm{E})\end{array}$ & $\begin{array}{c}\text { Water depth } \\
(\mathrm{m})\end{array}$ \\
\hline $752 \mathrm{~A}$ & $30^{\circ} 53.48^{\prime}$ & $93^{\circ} 34.65^{\prime}$ & 1086 \\
$754 \mathrm{~A}$ & $30^{\circ} 56.44^{\prime}$ & $93^{\circ} 33.99^{\prime}$ & 1064 \\
$756 \mathrm{~B}$ & $27^{\circ} 21.33^{\prime}$ & $87^{\circ} 35.80^{\prime}$ & 1518 \\
$756 \mathrm{C}$ & $27^{\circ} 21.25^{\prime}$ & $87^{\circ} 35.89^{\prime}$ & 1516 \\
$757 \mathrm{~B}$ & $1^{\circ} 01.46^{\prime}$ & $88^{\circ} 10.90^{\prime}$ & 1652 \\
\hline
\end{tabular}

\section{METHODOLOGY}

Samples entering our laboratory were freeze-dried, split, and then sieved and passed through $425-\mu \mathrm{m}, 150-\mu \mathrm{m}$, and $63-\mu \mathrm{m}$ sieves. The benthic foraminifer Cibicidoides $\mathrm{sp}$. was picked from the fraction coarser than $150 \mu \mathrm{m}$ from the Broken Ridge samples. At Sites 756 and 757 Cibicidoides sp. was not present throughout the core, so we picked Uvigerina sp. and Gyroidinoides sp. for our analyses.

All samples were roasted in vacuo at $380^{\circ} \mathrm{C}$ for $1 \mathrm{hr}$ to remove volatile organic contaminants. Foraminiferal samples of one to four or five individuals, ranging in weight from 50 to $300 \mu \mathrm{g}$ $\mathrm{CaCO}_{3}$ were individually reacted at $73^{\circ} \mathrm{C}$ with anhydrous phosphoric acid (density $1.90-1.92 \mathrm{~g} / \mathrm{cm}^{3}$ ) in an online extraction device (CarboKiel), which allows for the reaction of samples within separate reaction vessels. Four drops of acid were added to each vessel containing carbonate while under vacuum. Such a procedure eliminates progressive $\mathrm{CO}_{2}$ contamination inherent in common acid bath reaction systems; thus, samples as small as $\mathbf{5 0}$ $\mu \mathrm{g}$ can be sequentially analyzed without loss of analytical accuracy. Analyses were performed on a Finnegan MAT 251 mass spectrometer.

Isotopic enrichments were measured relative to a laboratory $\mathrm{CO}_{2}$ reference gas and corrected for ${ }^{17} \mathrm{O}$ contributions. Measured values were then converted to per mil enrichments relative to the PDB standard through calibration with NBS-20 powdered carbonate $\left(-4.14 \delta^{18} \mathrm{O}\right.$ and $-1.06 \delta^{13} \mathrm{C}$ published values). Precision of analysis (1-sigma) was calculated from daily analyses of NB-20 which bracketed and were intermixed within the sample analysis sequence (mean and 1-sigma values for the standard are $\delta^{13} \mathrm{C}=$ $\left.-1.03(0.07) ; \delta^{18} \mathrm{O}=-4.14(.07) ; n=261\right)$. Analyses were excluded only when machine operational errors were evident.

\section{OXYGEN AND CARBON ISOTOPE RECORD}

\section{Oxygen Isotopes}

The $\delta^{18} \mathrm{O}$ values from all four sites show the important Neogene changes in the isotopic chemistry of the oceans (Tables 2-6) The Cibicidoides record from Hole 752A (Fig. 2, Table 2) shows the heaviest values, averaging about 2.4 per mil in the uppermost $10 \mathrm{~m}$, values averaging about 1.6 to 1.8 per mil between 10 and $52 \mathrm{~m}$, a sharp decline to values averaging about 0.8 per mil (excluding the "peak" at $78 \mathrm{mbsf}$ ) that extends downcore to 95 mbsf, and the lowermost values decline to about -0.5 per mil. Hole 754A contains a very similar Cibicidoides record (Fig. 3, Table 3); the upper $10 \mathrm{~m}$ have average $\delta^{18} \mathrm{O}$ values of about 2.6 per mil. A transition between 10 and 18 mbsf occurs and from 18 to about $72 \mathrm{mbsf}$ the $\delta^{18} \mathrm{O}$ values average about 1.7 to 1.8 per mil. Between 72 and 78 mbsf a second transition occurs as values decline to an average of about 1 per mil in the lower portion of the core.

The records at Holes 756B, 756C, and 757B were obtained from different genera than those analyzed at the Broken Ridge sites. The record from Holes 756B and 756C (Figs. 4 and 5, Tables
Table 2. Oxygen and carbon isotopic data from Hole $752 \mathrm{~A}$.

\begin{tabular}{|c|c|c|c|}
\hline $\begin{array}{l}\text { Core, section, } \\
\text { interval }(\mathrm{cm})\end{array}$ & $\begin{array}{l}\text { Depth } \\
\text { (mbsf) }\end{array}$ & Oxygen-18 & Carbon-13 \\
\hline \multicolumn{4}{|l|}{$121.752 \mathrm{~A}-$} \\
\hline $1 \mathrm{H}-1,80-82$ & 0.80 & 2.26 & 0.99 \\
\hline $1 \mathrm{H}-2,80-82$ & 2.30 & 2.05 & 1.36 \\
\hline $1 \mathrm{H}-3,80-82$ & 3.80 & 2.27 & 1.32 \\
\hline $1 \mathrm{H}-4,80-82$ & 5.30 & 2.65 & 0.75 \\
\hline $1 \mathrm{H}-5,80-82$ & 6.80 & 2.38 & 0.84 \\
\hline $2 \mathrm{H}-1,80-82$ & 9.10 & 2.07 & 0.80 \\
\hline $2 \mathrm{H}-2,80-82$ & 10.60 & 1.92 & 0.92 \\
\hline $2 \mathrm{H}-3,80-82$ & 12.10 & 1.88 & 0.69 \\
\hline $2 \mathrm{H}-4,80-82$ & 13.60 & 1.72 & 0.52 \\
\hline $2 \mathrm{H}-5,80-82$ & 15.10 & 1.68 & 0.82 \\
\hline $2 \mathrm{H}-6,80-82$ & 16.60 & 1.89 & 0.76 \\
\hline $3 \mathrm{H}-1,80-82$ & 18.60 & 1.68 & 1.02 \\
\hline $3 \mathrm{H}-2,78-80$ & 20.08 & 1.86 & 0.85 \\
\hline $3 \mathrm{H}-3,80-82$ & 21.60 & 1.84 & 0.77 \\
\hline $3 \mathrm{H}-4,78-80$ & 23.08 & 1.79 & 1.01 \\
\hline $3 \mathrm{H}-5,80-82$ & 24.60 & 1.61 & 0.83 \\
\hline $3 \mathrm{H}-6,80-82$ & 26.10 & 1.79 & 1.43 \\
\hline $4 \mathrm{H}-1,80-82$ & 28.10 & 1.81 & 1.35 \\
\hline $4 \mathrm{H}-2,80-82$ & 29.60 & 1.78 & 1.28 \\
\hline $4 \mathrm{H}-3,80-82$ & 31.10 & 1.80 & 1.45 \\
\hline $4 \mathrm{H}-4,78-80$ & 32.58 & 1.55 & 1.01 \\
\hline $4 \mathrm{H}-5,80-82$ & 34.10 & 1.82 & 1.32 \\
\hline $4 \mathrm{H}-6,80-82$ & 35.60 & 1.69 & 1.24 \\
\hline $5 \mathrm{H}-1,85-87$ & 37.65 & 0.58 & 0.35 \\
\hline $5 \mathrm{H}-2,85-87$ & 39.15 & 1.54 & 1.36 \\
\hline $5 \mathrm{H}-3,85-87$ & 40.65 & 1.56 & 1.61 \\
\hline $5 \mathrm{H}-4,85-87$ & 42.15 & 1.65 & 1.33 \\
\hline $5 \mathrm{H}-5,85-87$ & 43.65 & 1.62 & 1.44 \\
\hline $5 \mathrm{H}-6,85-87$ & 45.15 & 1.61 & 1.57 \\
\hline $6 \mathrm{H}-1,85-87$ & 47.25 & 1.62 & 1.88 \\
\hline $6 \mathrm{H}-2,85-87$ & 48.75 & 1.68 & 1.46 \\
\hline $6 \mathrm{H}-3,85-87$ & 50.25 & 1.62 & 1.48 \\
\hline $6 \mathrm{H}-4,85-87$ & 51.75 & 1.63 & 1.70 \\
\hline $6 \mathrm{H}-5,85-87$ & 53.25 & 1.66 & 1.68 \\
\hline $7 \mathrm{H}-1,85-87$ & 56.95 & 1.34 & 1.87 \\
\hline $7 \mathrm{H}-2,85-87$ & 58.45 & 1.24 & 2.14 \\
\hline $7 \mathrm{H}-3,85-87$ & 59.95 & 1.08 & 1.95 \\
\hline $7 \mathrm{H}-4,85-87$ & 61.45 & 0.74 & 1.59 \\
\hline $7 \mathrm{H}-5,85-87$ & 62.95 & 1.14 & 2.20 \\
\hline $7 \mathrm{H}-6,85-87$ & 64.45 & 1.05 & 2.03 \\
\hline $8 \mathrm{H}-1,85-87$ & 66.65 & 0.77 & 1.71 \\
\hline $8 \mathrm{H}-2,85-87$ & 68.15 & 0.62 & 1.26 \\
\hline $8 \mathrm{H}-3,85-87$ & 69.65 & 0.38 & 1.38 \\
\hline $8 \mathrm{H}-4,85-87$ & 71.15 & 0.73 & 1.69 \\
\hline $8 \mathrm{H}-5,85-87$ & 72.65 & 1.03 & 1.58 \\
\hline $8 \mathrm{H}-6,85-87$ & 74.15 & 0.78 & 1.06 \\
\hline $9 \mathrm{H}-1,85-87$ & 76.25 & 2.76 & 0.95 \\
\hline $9 \mathrm{H}-2,85-87$ & 77.75 & 1.66 & 0.96 \\
\hline $9 \mathrm{H}-3,85-87$ & 79.25 & 1.03 & 1.39 \\
\hline $9 \mathrm{H}-4,85-87$ & 80.75 & 1.10 & 1.26 \\
\hline $9 \mathrm{H}-5,85-87$ & 82.25 & 0.92 & 1.36 \\
\hline $9 \mathrm{H}-6,85-87$ & 83.75 & 0.70 & 1.29 \\
\hline $10 \mathrm{H}-1,85-87$ & 85.85 & 1.08 & 1.42 \\
\hline $10 \mathrm{H}-3,85-87$ & 88.85 & 0.07 & 1.11 \\
\hline $10 \mathrm{H}-4,85-87$ & 90.35 & 1.24 & 1.80 \\
\hline $10 \mathrm{H}-5,85-87$ & 91.85 & 0.93 & 1.72 \\
\hline $10 \mathrm{H}-6,85-87$ & 93.40 & 0.76 & 1.33 \\
\hline $11 \mathrm{H}-1,85-87$ & 95.55 & 0.26 & 1.37 \\
\hline $11 \mathrm{H}-2,90-92$ & 97.10 & 0.10 & 1.41 \\
\hline $11 \mathrm{H}-3,85-87$ & 98.55 & 0.16 & 1.31 \\
\hline $11 \mathrm{H}-4,85-87$ & 100.05 & -0.53 & 1.64 \\
\hline $11 \mathrm{H}-5,85-87$ & 101.55 & -0.24 & 1.83 \\
\hline $11 \mathrm{H}-5,135-137$ & 102.05 & -0.45 & 1.74 \\
\hline
\end{tabular}

Note: All analyses were conducted on the benthic foraminifer Cibicidoides sp. 
Table 3. Oxygen and carbon isotopic data from Hole 754A.

\begin{tabular}{|c|c|c|c|}
\hline $\begin{array}{l}\text { Core, section } \\
\text { interval }(\mathrm{cm})\end{array}$ & $\begin{array}{l}\text { Depth } \\
\text { (mbsf) }\end{array}$ & Oxygen-18 & Carbon-13 \\
\hline \multicolumn{4}{|l|}{$121-754 \mathrm{~A}-$} \\
\hline $1 \mathrm{H}-1,78-80$ & 0.78 & 2.72 & 0.94 \\
\hline $1 \mathrm{H}-2,78-80$ & 2.28 & 2.57 & 1.03 \\
\hline $1 \mathrm{H}-3,78-80$ & 3.78 & 2.58 & 0.96 \\
\hline $1 \mathrm{H}-4,78-80$ & 5.28 & 2.52 & 1.11 \\
\hline $2 \mathrm{H}-1,78-80$ & 6.88 & 2.67 & 1.06 \\
\hline $2 \mathrm{H}-2,78-80$ & 8.38 & 2.62 & 1.07 \\
\hline $2 \mathrm{H}-3,78-80$ & 9.88 & 2.51 & 0.95 \\
\hline $2 \mathrm{H}-4,78-80$ & 11.38 & 2.46 & 0.77 \\
\hline $2 \mathrm{H}-5,78-80$ & 12.88 & 2.34 & 0.83 \\
\hline $2 \mathrm{H}-6,78-80$ & 14.38 & 2.23 & 0.80 \\
\hline $3 \mathrm{H}-1,78-80$ & 16.48 & 2.12 & 0.70 \\
\hline $3 \mathrm{H}-2,78-80$ & 17.98 & 2.03 & 0.70 \\
\hline $3 \mathrm{H}-3,78-80$ & 19.48 & 1.77 & 1.00 \\
\hline $3 \mathrm{H}-4,78-80$ & 20.98 & 1.79 & 0.70 \\
\hline $3 \mathrm{H}-5,78-80$ & 22.48 & 2.07 & 0.75 \\
\hline $3 \mathrm{H}-6,78-80$ & 23.98 & 2.21 & 0.88 \\
\hline $4 \mathrm{H}-1,78-80$ & 26.08 & 2.06 & 0.87 \\
\hline $4 \mathrm{H}-2,78-80$ & 27.58 & 1.93 & 0.79 \\
\hline $4 \mathrm{H}-3,78-80$ & 29.08 & 2.07 & 1.03 \\
\hline $4 \mathrm{H}-4,78-80$ & 30.58 & 1.75 & 0.87 \\
\hline $4 \mathrm{H}-5,78-80$ & 32.08 & 1.84 & 1.11 \\
\hline $4 \mathrm{H}-6,78-80$ & 33.58 & 1.81 & 1.24 \\
\hline $5 \mathrm{H}-1,78-80$ & 35.68 & 1.98 & 1.34 \\
\hline $5 \mathrm{H}-2,78-80$ & 37.18 & 1.81 & 1.37 \\
\hline $5 \mathrm{H}-3,78-80$ & 38.68 & 2.04 & 1.13 \\
\hline $5 \mathrm{H}-4,78-80$ & 40.18 & 1.74 & 1.48 \\
\hline $5 \mathrm{H}-5,78-80$ & 41.68 & 1.79 & 1.33 \\
\hline $5 \mathrm{H}-6,78-80$ & 43.18 & 1.78 & 1.45 \\
\hline $6 \mathrm{H}-1,78-80$ & 45.28 & 2.10 & 1.17 \\
\hline $6 \mathrm{H}-2,78-80$ & 46.78 & 2.06 & 0.82 \\
\hline $6 \mathrm{H}-3,78-80$ & 48.28 & 1.86 & 1.16 \\
\hline $6 \mathrm{H}-4,78-80$ & 49.78 & 1.57 & 1.10 \\
\hline $6 \mathrm{H}-5,78-80$ & 51.28 & 1.82 & 1.16 \\
\hline $6 \mathrm{H}-6,78-80$ & 52.78 & 1.85 & 1.44 \\
\hline $7 \mathrm{H}-1,78-80$ & 54.88 & 1.56 & 1.34 \\
\hline $7 \mathrm{H}-2,78-80$ & 56.38 & 1.65 & 1.08 \\
\hline $7 \mathrm{H}-3,78-80$ & 57.88 & 1.77 & 1.55 \\
\hline $7 \mathrm{H}-4,78-80$ & 59.38 & 1.74 & 1.48 \\
\hline $7 \mathrm{H}-5,78-80$ & 60.88 & 1.87 & 1.96 \\
\hline $7 \mathrm{H}-6,78-80$ & 62.38 & 1.62 & 1.51 \\
\hline $8 \mathrm{H}-1,78-80$ & 64.58 & 1.54 & 1.63 \\
\hline
\end{tabular}

4 and 5) show the uppermost few samples to have average $\delta^{18} \mathrm{O}$ values of about 3 per mil, a sequence of values near 2.5 per mil, and a transition between about 42 and $52 \mathrm{mbsf}$ to values averaging 1.5 to 1.7 per mil. The lowest $15 \mathrm{~m}$ (in Hole $756 \mathrm{C}$ ) are characterized by the lightest values, about 0.4 per mil for the Uvigerinids and 0.8 per mil for the Gyroidinoidids. The oxygen isotopic record from Hole 757B (Fig. 6, Table 6) is similar, exhibiting values in excess of 3 per mil in the upper $15 \mathrm{~m}$, a series of values averaging 2.7 per mil, a transition at approximately 85 mbsf to values of 1.8 to 2.0 per mil, and a decline at about $120 \mathrm{mbsf}$ to values of about 1.2 per mil in the lower portion of the measured section.

We can use the nannofossil zonations provided by Peirce, Weissel, et al. (1989), with augmentation by P. Resiwati (pers. comm., 1990), to determine when the transitions in isotopic values occurred. The lowest transition, best illustrated by the data from Sites 756 and 757, occurs at the Eocene/Oligocene boundary. At Site 756 this transition is marked by an overall enrichment of nearly 1.2 per mil in the Uvigerinid data and of about 1 per mil in the Gyroidinoidid data. At Site 757 the enrichments are similar. This increase of about 1 per mil represents the cooling of the Southern Ocean, formation of cold bottom water, and the creation of some to much of the ice on Antarctica (Kennett and Shackleton, 1976; Kennett, 1977; Matthews and Poore, 1980). The paleodepth of Sites 756 and 757 at the time of the Eocene/Oligocene boundary
Table 3 (Continued).

\begin{tabular}{|c|c|c|c|}
\hline $\begin{array}{l}\text { Core, section } \\
\text { interval }(\mathrm{cm})\end{array}$ & $\begin{array}{l}\text { Depth } \\
\text { (mbsf) }\end{array}$ & Oxygen-18 & Carbon-13 \\
\hline \multicolumn{4}{|l|}{ 121-754A-(Cont.) } \\
\hline $8 \mathrm{H}-2,78-80$ & 66.08 & 1.92 & 1.75 \\
\hline $8 \mathrm{H}-3,78-80$ & 67.58 & 1.69 & 1.74 \\
\hline $8 \mathrm{H}-4,78-80$ & 69.08 & 1.68 & 1.81 \\
\hline $8 \mathrm{H}-5,78-80$ & 70.58 & 1.67 & 1.69 \\
\hline $8 \mathrm{H}-6,78-80$ & 72.08 & 1.58 & 1.74 \\
\hline $8 \mathrm{H}-7,78-80$ & 73.58 & 1.60 & 1.83 \\
\hline $9 \mathrm{H}-1,78-80$ & 74.28 & 1.23 & 1.84 \\
\hline $9 \mathrm{H}-2,78-80$ & 75.78 & 1.24 & 1.95 \\
\hline $9 \mathrm{H}-3,78-80$ & 77.28 & 0.80 & 2.00 \\
\hline $9 \mathrm{H}-4,78-80$ & 78.78 & 0.99 & 2.26 \\
\hline $9 \mathrm{H}-5,78-80$ & 80.28 & 0.87 & 1.91 \\
\hline $9 \mathrm{H}-6,78-80$ & 81.78 & 1.29 & 2.41 \\
\hline $10 \mathrm{H}-1,78-80$ & 83.98 & 0.82 & 1.58 \\
\hline $10 \mathrm{H}-2,78-80$ & 85.48 & 1.23 & 1.51 \\
\hline $10 \mathrm{H}-3,78-80$ & 86.98 & 0.91 & 0.93 \\
\hline $10 \mathrm{H}-4,78-80$ & 88.48 & 1.16 & 1.43 \\
\hline $10 \mathrm{H}-5,78-80$ & 89.98 & 1.02 & 1.35 \\
\hline $10 \mathrm{H}-6,78-80$ & 91.48 & 0.96 & 1.50 \\
\hline $11 \mathrm{H}-1,78-80$ & 93.68 & 1.25 & 1.27 \\
\hline $11 \mathrm{H}-2,78-80$ & 95,18 & 1.23 & 1.49 \\
\hline $11 \mathrm{H}-3,78-80$ & 96.68 & 1.11 & 1.38 \\
\hline $11 \mathrm{H}-4,80-82$ & 98.2 & 1.28 & 1.45 \\
\hline $11 \mathrm{H}-5,78-80$ & 99.68 & 0.88 & 1.40 \\
\hline $11 \mathrm{H}-6,78-80$ & 101.18 & 0.97 & 1.50 \\
\hline $12 \mathrm{H}-1,78-80$ & 103.38 & 1.29 & 1.69 \\
\hline $12 \mathrm{H}-2,78-80$ & 104.88 & 1.20 & 1.79 \\
\hline $12 \mathrm{H}-3,78-80$ & 106.38 & 1.24 & 1.40 \\
\hline $12 \mathrm{H}-4,78-80$ & 107.88 & 0.67 & 1.24 \\
\hline $12 \mathrm{H}-5,78-80$ & 109.38 & 0.79 & 1.26 \\
\hline $12 \mathrm{H}-6,78-80$ & 110.88 & 1.11 & 1.23 \\
\hline $13 \mathrm{H}-1,78-80$ & 113.08 & 1.08 & 0.78 \\
\hline $13 \mathrm{H}-2,78-80$ & 114.58 & 1.06 & 1.22 \\
\hline $13 \mathrm{H}-3,78-80$ & 116.08 & 1.06 & 1.05 \\
\hline $14 \mathrm{H}-1,78-80$ & 122.78 & 0.60 & 1.52 \\
\hline $14 \mathrm{H}-2,78-80$ & 124.28 & 0.18 & 1.75 \\
\hline $14 \mathrm{H}-3,78-80$ & 125.78 & 1.65 & 1.46 \\
\hline
\end{tabular}

Note: All analyses were conducted on the benthic foraminifer Cibicidoides sp.

was within the Surface Water Mass, so the $\delta^{18} \mathrm{O}$ shift recorded here is not a record of deep-ocean temperature; it may reflect either the whole-ocean response to ice formation, a sudden cooling of the surface waters at the paleolatitude of these sites, or a combination of effects. The isotopically light values from the lower samples of Holes $752 \mathrm{~A}$ and $754 \mathrm{~A}$ are also from Eocene sediments deposited above the Broken Ridge angular unconformity.

The middle Miocene oxygen isotopic enrichment occurs mostly in Zone CN5a, 14.4 to 13.0 Ma (Berggren et al., 1985), on Broken Ridge and Ninetyeast Ridge. This enrichment is about 0.8 to 1.0 per mil and reflects additional ice buildup on Antarctica, possibly associated with additional polar cooling (Kennett, 1977, 1985; Woodruff et al., 1981). Mid-Miocene depths of these drill sites would place them within the Surface Water Mass then.

The upper Pliocene oxygen isotopic enrichment occurs in Zones CN11 and CN12, 3.6 to 2.0 Ma (Berggren et al., 1985), and is 0.7 to 0.8 per mil in these sites. It reflects the oceanic response to the formation of Northern Hemisphere ice.

\section{Carbon Isotopes}

The $\delta^{13} \mathrm{C}$ record from Hole 752A (Fig. 2, Table 2) on Broken Ridge decreases from core-top values to a low of about 0.5 per mil near 14 mbsf. From that low, values increase downcore to a maximum at roughly $60 \mathrm{mbsf}$; an important increase of about 0.7 per mil occurs at 27 mbsf. At about $62 \mathrm{mbsf}$, the $\delta^{13} \mathrm{C}$ values 
Table 4. Oxygen and carbon isotopic data from Hole 756B.

\begin{tabular}{|c|c|c|c|c|c|}
\hline $\begin{array}{l}\text { Core, section } \\
\text { interval }(\mathrm{cm})\end{array}$ & $\begin{array}{l}\text { Depth } \\
\text { (mbsf) }\end{array}$ & $\begin{array}{c}\text { Gyroidinoides sp. } \\
\text { Oxygen-18 }\end{array}$ & $\begin{array}{l}\text { Gyroidinoides sp. } \\
\text { Carbon-13 }\end{array}$ & $\begin{array}{c}\text { Uvigerina sp. } \\
\text { Oxygen-18 }\end{array}$ & $\begin{array}{l}\text { Uvigerina sp. } \\
\text { Carbon- } 13\end{array}$ \\
\hline \multicolumn{6}{|l|}{$121-756 \mathrm{~B}-$} \\
\hline $1 \mathrm{H}-1,80-82$ & 0.80 & & 3.12 & 0.32 & \\
\hline $1 \mathrm{H}-2,80-82$ & 2.30 & & 3.50 & -0.02 & \\
\hline $1 \mathrm{H}-3,80-82$ & 3.80 & & 2.38 & -0.08 & \\
\hline $1 \mathrm{H}-4,80-82$ & 5.30 & & 3.04 & -0.04 & \\
\hline IH $-5,80-82$ & 6.80 & & 2.24 & 0.78 & \\
\hline $2 \mathrm{H}-\mathrm{I}, 80-82$ & 9.30 & & 2.80 & 0.25 & \\
\hline $2 \mathrm{H}-2,80-82$ & 10.80 & & 2.72 & 0.53 & \\
\hline $2 \mathrm{H}-3,80-82$ & 12.30 & & 2.65 & 0.36 & \\
\hline $2 \mathrm{H}-4,80-82$ & 13.80 & & 2.78 & 0.25 & \\
\hline $2 \mathrm{H}-5,80-82$ & 15.30 & & 2.73 & 0.17 & \\
\hline $2 \mathrm{H}-6,80-82$ & 16.80 & & 2.71 & 0.49 & \\
\hline $3 \mathrm{H}-1,80-82$ & 18.90 & 2.94 & -0.03 & 2.21 & 0.20 \\
\hline $3 \mathrm{H}-2,80-82$ & 20.40 & & 2.79 & 0.32 & \\
\hline $3 \mathrm{H}-3,80-82$ & 21.90 & & 2.27 & 0.13 & \\
\hline $3 \mathrm{H}-4,80-82$ & 23.40 & & 2.68 & 0.27 & \\
\hline $3 \mathrm{H}-5,80-82$ & 24.90 & & 2.45 & 0.32 & \\
\hline $3 \mathrm{H}-6,80-82$ & 26.40 & 2.36 & 0.33 & 2.40 & 0.56 \\
\hline $4 \mathrm{H}-1,80-82$ & 28.50 & 2.77 & 0.72 & 2.49 & 0.72 \\
\hline $4 \mathrm{H}-3,80-82$ & 31.50 & & 2.60 & 0.91 & \\
\hline $4 \mathrm{H}-5,80-82$ & 34.50 & & 2.49 & 0.64 & \\
\hline $4 \mathrm{H}-6,80-82$ & 36.00 & & 2.52 & 1.01 & \\
\hline $5 \mathrm{H}-1,80-82$ & 38.10 & & 2.34 & 0.66 & \\
\hline $5 \mathrm{H}-2,80-82$ & 39.60 & & 2.39 & 0.77 & \\
\hline $5 \mathrm{H}-4,80-82$ & 42.60 & 2.48 & 0.55 & 2.18 & 1.09 \\
\hline $5 \mathrm{H}-5,80-82$ & 44.10 & & 2.23 & 0.93 & \\
\hline $5 \mathrm{H}-6,80-82$ & 45.60 & 1.78 & 0.80 & & \\
\hline $6 \mathrm{H}-2,80-82$ & 49.20 & 1.70 & 1.34 & & \\
\hline $6 \mathrm{H}-3,80-82$ & 50.70 & & 1.10 & 1.76 & \\
\hline $6 \mathrm{H}-5,80-82$ & 53.70 & 1.24 & 0.47 & 1.38 & 1.39 \\
\hline $7 \mathrm{H}-1,80-82$ & 57.00 & 1.64 & 0.50 & 1.18 & 1.06 \\
\hline $7 \mathrm{H}-3,80-82$ & 60.00 & 1.89 & 0.96 & & \\
\hline $7 \mathrm{H}-4,80-82$ & 61.50 & & 1.80 & 1.01 & \\
\hline $7 \mathrm{H}-5,84-86$ & 63.04 & 1.74 & 0.99 & 1.41 & 0.80 \\
\hline $7 \mathrm{H}-6,80-82$ & 64.50 & & 1.73 & 1.28 & \\
\hline $8 \mathrm{H}-1,80-82$ & 66.40 & 1.84 & 1.26 & 1.62 & 1.20 \\
\hline $8 \mathrm{H}-2,80-82$ & 67.90 & 1.95 & 1.31 & & \\
\hline $8 \mathrm{H}-3,80-82$ & 69.40 & 1.68 & 1.00 & 1.59 & 1.19 \\
\hline $8 \mathrm{H}-4,80-82$ & 70.90 & 1.54 & 0.54 & 1.41 & 0.66 \\
\hline $8 \mathrm{H}-5,80-82$ & 72.40 & 1.57 & 0.64 & & \\
\hline $8 \mathrm{H}-6,80-82$ & 73.90 & 1.64 & 0.63 & & \\
\hline $9 \mathrm{H}-1,80-82$ & 76.00 & 1.62 & 0.42 & 1.77 & 0.73 \\
\hline $9 \mathrm{H}-2,80-82$ & 77.50 & 1.86 & 0.67 & 1.38 & 0.42 \\
\hline $9 \mathrm{H}-3,80-82$ & 79.00 & 1.82 & 0.26 & & \\
\hline $9 \mathrm{H}-4,80-82$ & 80.50 & & 1.36 & 0.29 & \\
\hline $9 \mathrm{H}-5,80-82$ & 82.00 & 1.71 & 0.36 & & \\
\hline $9 \mathrm{H}-6,80-82$ & 83.50 & 1.77 & 0.45 & 1.62 & 0.46 \\
\hline $10 \mathrm{H}-\mathrm{I}, 80-82$ & 85.70 & 1.73 & 0.54 & 1.56 & 0.65 \\
\hline $10 \mathrm{H}-2,80-82$ & 87.20 & 1.77 & 0.52 & 1.47 & 0.55 \\
\hline $10 \mathrm{H}-3,30-32$ & 88.20 & 1.65 & 0.46 & & \\
\hline $10 \mathrm{H}-3,80-82$ & 88.70 & & 1.38 & 0.66 & \\
\hline $10 \mathrm{H}-4,80-82$ & 90.20 & 1.54 & 0.57 & 1.44 & 0.47 \\
\hline $10 \mathrm{H}-5,80-82$ & 91.70 & 1.65 & 0.21 & 1.29 & 0.35 \\
\hline $10 \mathrm{H}-6,80-82$ & 93.20 & & & & \\
\hline $1 \mathrm{H}-1,80-82$ & 95.40 & 1.55 & 0.94 & & \\
\hline $11 \mathrm{H}-2,80-82$ & 96.90 & & 1.60 & 1.18 & \\
\hline $11 \mathrm{H}-3,80-82$ & 98.40 & 1.20 & 0.77 & & \\
\hline $11 \mathrm{H}-4,80-82$ & 99.90 & 1.16 & 0.63 & 1.34 & 0.73 \\
\hline $11 \mathrm{H}-5,80-82$ & 101.40 & & 1.14 & 0.72 & \\
\hline $11 \mathrm{H}-6,80-82$ & 102.90 & & 1.37 & 1.17 & \\
\hline
\end{tabular}

Note: Analyses were conducted on the benthic foraminifers Gyroidinoides sp. and Uvigerina sp.

decline by approximately 0.8 per mil to values averaging about 1.4 per mil in the lower portion of the sequence. Site 754 contains a similar record (Fig. 3, Table 3). Values decline gently from the top of the core to a low of about 1.7 per mil near 20 mbsf, and then increase to a maximum of 2.5 per mil near $80 \mathrm{mbsf}$. Approximately 0.6 per mil of this increase occurs near 35 mbsf. Between 82 and 87 mbsf the $\delta^{13} \mathrm{C}$ values decline nearly 0.9 per mil to values of about 1.4 per mil that characterize the lower portion of the core.
At Site 756 the same aspects of the $\delta^{13} \mathrm{C}$ record occur (Figs. 4 and 5, Tables 4 and 5), with low values near the top, increasing downcore to a maximum of 1.8 per mil at about $52 \mathrm{mbsf}$, and an increase of 0.7 per mil centered near 27 mbsf. Between about 52 and $57 \mathrm{mbsf}$ the carbon isotopic values decline by about 0.8 per mil and fluctuate around values of 0.5 to 1.0 per mil through the lower half of the record. Both genera show very similar $\delta^{13} \mathrm{C}$ fluctuations in the lower $80 \mathrm{~m}$ at Site 756 , and record a modest 
Table 5. Oxygen and carbon isotopic data from Hole 756C.

\begin{tabular}{lccccc}
\hline $\begin{array}{c}\text { Core, section } \\
\text { interval }(\mathrm{cm})\end{array}$ & $\begin{array}{c}\text { Depth } \\
\text { (mbsf) }\end{array}$ & $\begin{array}{c}\text { Gyroidinoides sp. } \\
\text { Oxygen-18 }\end{array}$ & $\begin{array}{c}\text { Gyroidinoides sp. } \\
\text { Carbon-13 }\end{array}$ & $\begin{array}{c}\text { Uvigerina sp. } \\
\text { Oxygen-18 }\end{array}$ & $\begin{array}{c}\text { Uvigerina sp. } \\
\text { Carbon-13 }\end{array}$ \\
\hline 121-756C- & & & & & \\
4X-1, 80-82 & 101.70 & 1.76 & 0.35 & 1.72 & 0.60 \\
$4 \mathrm{X}-2,80-82$ & 103.20 & 1.76 & 0.64 & 1.52 & 0.49 \\
$4 \mathrm{X}-3,80-82$ & 104.70 & 1.66 & 0.31 & 1.35 & 0.39 \\
$4 \mathrm{X}-4,80-82$ & 106.20 & 1.73 & 0.40 & 1.69 & 0.34 \\
$4 \mathrm{X}-5,80-82$ & 107.20 & 1.44 & 0.52 & & 1.15 \\
$4 \mathrm{X}-6,80-82$ & 109.20 & 1.64 & 0.49 & 2.64 & 0.26 \\
$5 \mathrm{X}-3,80-82$ & 112.32 & 1.57 & 0.55 & 1.24 & 1.04 \\
$5 \mathrm{X}-5,80-82$ & 114.26 & 1.45 & 0.62 & & \\
$5 \mathrm{X}-6,80-82$ & 115.08 & 1.56 & 0.89 & 0.67 & 1.15 \\
$5 \mathrm{X}-7,80-82$ & 116.41 & 1.41 & 0.86 & & 1.55 \\
$5 \mathrm{X}-8,80-82$ & 117.91 & 1.13 & 0.58 & 1.41 & 0.85 \\
$6 \mathrm{X}-1,80-82$ & 121.00 & 1.60 & 1.14 & 1.38 & 0.79 \\
$6 \mathrm{X}-2,80-82$ & 122.50 & 1.69 & 1.37 & 0.72 & \\
$6 \mathrm{X}-4,80-82$ & 125.50 & 1.08 & 1.02 & 0.46 & \\
$6 \mathrm{X}-5,80-82$ & 127.00 & 1.02 & 0.67 & 0.48 & 1.41 \\
$7 \mathrm{X}-1,80-82$ & 130.60 & 1.10 & 1.13 & 0.96 & \\
$7 \mathrm{X}-2,80-82$ & 132.10 & & 0.65 & 1.07 & \\
$7 \mathrm{X}-3,80-82$ & 133.60 & & 0.58 & 0.54 & \\
$7 \mathrm{X}-4,80-82$ & 135.10 & 0.65 & 1.18 & 0.34 & \\
$7 \mathrm{X}-5,80-82$ & 136.60 & 0.89 & 0.79 & 0.83 & \\
$7 \mathrm{X}-6,80-82$ & 138.10 & & 0.39 & & \\
$8 \mathrm{X}-1,80-82$ & 140.20 & & 0.53 & & \\
\hline
\end{tabular}

Note: Analyses were conducted on the benthic foraminifers Gyroidinoides sp. and Uvigerina sp.

decrease of perhaps 0.5 per mil in $\delta^{13} \mathrm{C}$ at 68 to $75 \mathrm{mbsf}$ and a similar increase at 112 to $122 \mathrm{mbsf}$. Carbon isotopic values at Hole 757B (Fig. 6, Table 6) display a general downcore increase in $\delta^{13} \mathrm{C}$ values, with the largest step at 51 to $57 \mathrm{mbsf}$.

The lower enrichment of $\delta^{13} \mathrm{C}$ occurs in nannofossil zone CN4 at Sites 752 and 754 and within Zones CN2 to CN4 at Site 756. This enrichment of 0.8 or 0.9 per mil in $\delta^{13} \mathrm{C}$ values that occurred about 15 to $17 \mathrm{Ma}$ occurs in both planktonic and benthic foraminifers and thus represents oceanic exchange with a marine or terrestrial sedimentary reservoir. Carbon storage on land or in marine sediments removes isotopically light organic carbon from the ocean, resulting in an increase in marine $\delta^{13} \mathrm{C}$; this inter-reservoir transfer is similar in concept to the effect of the storage of isotopically light ice away from the marine system and its effect on oxygen isotopes. This early/middle Miocene carbon isotopic shift coincides with a period of generally high sea-level (Haq et al., 1987) and the deposition of large amounts of organic carbon in basins around the North Pacific (the Chron 16 carbon shift, Vincent et al., 1985; Monterey event, Berger and Vincent, 1986).

The late Miocene depletion of 0.7 per mil in $\delta^{13} \mathrm{C}$ values occurs in Zone CN9 at Sites 754, 756, and 757, and within Zones CN9b to CN10a at Site 752. This depletion occurs throughout the oceans (Keigwin, 1979; Vincent et al., 1985; Kennett, 1985; Berger and Vincent, 1986; Keigwin et al., 1987) and has been demonstrated to occur within magnetic reversal Chron 6 (upper Zones CN9a and CN9b) at about 6.2 Ma (Keigwin and Shackleton, 1980).

\section{SUMMARY}

Oxygen and carbon stable isotopic records from the carbonate oozes at Sites $752,754,756$, and 757 provide the fundamental isotopic stratigraphy for these sediments. Oxygen isotopes provide evidence for cooling and/or ice volume increases at the Eocene/Oligocene boundary, in the middle Miocene, and in the late Pliocene. The carbon isotopic record contains the Chron 16 enrichment in the early/middle Miocene and the Chron 6 depletion in the latest Miocene.

\section{ACKNOWLEDGMENTS}

Lloyd Keigwin and an anonymous reviewer provided comments which served to improve this report. This work was supported by JOI-USSAC funding and by NSF grant OCE-8811299 to D. Rea.

\section{REFERENCES}

Berger, W. H., and Vincent, E., 1986. Deep-sea carbonates: reading the carbon-isotope signal. Geol. Rundsch., 75:249-269.

Berggren, W. A., Kent, D. V., Flynn, J. J., and Van Couvering, J. A., 1985. Cenozoic geochronology. Geol. Soc. Am. Bull., 96:1407-1418.

Bolli, H. M., Saunders, J. B., and Perch-Nielsen, K. (Eds.), 1985. Plankton Stratigraphy: Cambridge (Cambridge Univ. Press).

Davies, T. A., Luyendyk, B. P., et al., 1974. Init. Repts., DSDP, 26: Washington (U.S. Govt. Printing Office).

Haq, B. U., Hardenbol, J., and Vail, P. R., 1987. Chronology of fluctuating sea levels since the Triassic. Science, 235:1156-1167.

Keigwin, L. D., Aubry, M.-P., and Kent, D. V., 1987. North Atlantic late Miocene stable-isotope stratigraphy, biostratigraphy, and magnetostratigraphy. In Ruddiman, W. F., Kidd, R. B., et al., Init. Repts. DSDP, 94: Washington (U.S. Govt. Printing Office), 935-963.

Keigwin, L. D., Jr., 1979. Late Cenozoic stable isotope stratigraphy and paleoceanography of DSDP sites from the east equatorial and central North Pacific Ocean. Earth Planet. Sci. Lett., 45:361-381.

Keigwin, L. D., Jr., and Shackleton, N. J., 1980. Uppermost Miocene carbon isotope stratigraphy of a piston core in the equatorial Pacific. Nature, 284:613-614.

Kennett, J. P., 1977. Cenozoic evolution of Antarctic glaciation, the circum-Antarctic Ocean, and their impact on global paleoceanography. J. Geophys. Res., 82:3843-3860.

1986. Miocene to early Pliocene oxygen and carbon isotope stratigraphy of the Southwest Pacific, DSDP Leg 90. In Kennett, J. P., von der Borch, C. C., et al., Init. Repts. DSDP, 90 (Pt. 2): Washington (U.S. Govt. Printing Office), 1383-1411.

Kennett, J. P., and Shackleton, N. J., 1976. Oxygen isotopic evidence for the development of the psychrosphere 38 Myr ago. Nature, 260:513515.

Matthews, R. K., and Poore, R. Z., 1980. Tertiary $\delta^{18} \mathrm{O}$ record and glacio-eustatic sea-level fluctuations. Geology, 8:501-504. 
Peirce, J., Weissel, J., et al., 1989. Proc. ODP, Init. Repts., 121: College Station, TX (Ocean Drilling Program).

Rea, D. K., Dehn, J., Driscoll, N., Farrell, J., Janecek, T., Owen, R. M., Pospichal, J. L., Resiwati, P., and the ODP Leg 121 Scientific Party, 1990. Paleoceanography of the eastern Indian Ocean from ODP Leg 121 drilling on Broken Ridge. Geol. Soc. Am. Bull., 102:679-690.

Vincent, E., Killingley, J. S., and Berger, W. H., 1985. Miocene oxygen and carbon isotope stratigraphy of the tropical Indian Ocean. In Kennett, J. P. (Ed.), The Miocene Ocean: Paleoceanography and Biogeography. Mem. Geol. Soc. Am., 163:103-130.
Woodruff, F., Savin, S. M., and Douglas, R. G., 1981. Miocene stable isotope record: a detailed deep Pacific Ocean study and its paleoclimatic implications. Science, 212:665-668.

Date of initial receipt: 1 March 1990

Date of acceptance: 1 August 1990

Ms 121B-130 
Table 6. Oxygen and carbon isotopic data from Hole 757B.

\begin{tabular}{|c|c|c|c|c|c|}
\hline $\begin{array}{l}\text { Core, section } \\
\text { interval }(\mathrm{cm})\end{array}$ & $\begin{array}{l}\text { Depth } \\
\text { (mbsf) }\end{array}$ & $\begin{array}{l}\text { Uvigerina sp. } \\
\text { Oxygen-18 }\end{array}$ & $\begin{array}{l}\text { Uvigerina sp. } \\
\text { Carbon-13 }\end{array}$ & $\begin{array}{c}\text { Gyroidinoides sp. } \\
\text { Oxygen-18 }\end{array}$ & $\begin{array}{l}\text { Gyroidinoides sp. } \\
\text { Carbon- } 13\end{array}$ \\
\hline \multicolumn{6}{|l|}{$121-757 \mathrm{~B}-$} \\
\hline $1 \mathrm{H}-2,80-82$ & 2.13 & 3.01 & -0.09 & & \\
\hline $1 \mathrm{H}-3,80-82$ & 3.63 & 3.22 & -0.01 & & \\
\hline $2 \mathrm{H}-1,80-82$ & 5.30 & 3.37 & -0.38 & & \\
\hline $2 \mathrm{H}-2,80-82$ & 6.80 & 3.77 & -0.56 & & \\
\hline $2 \mathrm{H}-3,80-82$ & 8.30 & 3.02 & -0.02 & 3.33 & -0.36 \\
\hline $2 \mathrm{H}-4,80-82$ & 9.80 & 3.30 & -0.19 & & \\
\hline $2 \mathrm{H}-5,80-82$ & 11.30 & 3.30 & -0.27 & & \\
\hline $2 \mathrm{H}-6,80-82$ & 12.80 & 3.17 & 0.10 & & \\
\hline $3 \mathrm{H}-1,80-82$ & 14.80 & 3.27 & -0.07 & & \\
\hline $3 \mathrm{H}-2,80-82$ & 16.30 & 2.81 & 0.11 & & \\
\hline $3 \mathrm{H}-3,80-82$ & 17.80 & 2.83 & -0.14 & & \\
\hline $3 \mathrm{H}-4,80-82$ & 19.30 & 2.84 & 0.35 & & \\
\hline $3 \mathrm{H}-5,80-82$ & 20.80 & 3.07 & -0.17 & & \\
\hline $3 \mathrm{H}-6,80-82$ & 22.30 & 2.56 & -0.15 & & \\
\hline $4 \mathrm{H}-1,80-82$ & 24.40 & 2.81 & 0.06 & & \\
\hline $4 \mathrm{H}-2,80-82$ & 25.90 & & 2.97 & -0.48 & \\
\hline $4 \mathrm{H} \cdot 4,80-82$ & 28.90 & 2.62 & -0.06 & & \\
\hline $4 \mathrm{H}-5,80-82$ & 30.40 & 2.67 & 0.31 & & \\
\hline $5 \mathrm{H}-1,80-82$ & 34.00 & 2.87 & 0.12 & & \\
\hline $5 \mathrm{H}-2,80-82$ & 35.50 & 2.74 & -0.02 & 2.74 & -0.60 \\
\hline $5 \mathrm{H}-3,80-82$ & 37.00 & 2.55 & 0.21 & & \\
\hline $5 \mathrm{H}-4,80-82$ & 38.50 & 2.76 & 0.07 & & \\
\hline $5 \mathrm{H}-5,80-82$ & 40.00 & 2.68 & 0.28 & 2.79 & -0.08 \\
\hline $6 \mathrm{H}-1,80-82$ & 43.60 & 2.85 & 0.18 & 2.79 & -0.34 \\
\hline $6 \mathrm{H}-2,80-82$ & 45.10 & 2.66 & 0.25 & 2.72 & -0.28 \\
\hline $6 \mathrm{H}-3,80-82$ & 46.60 & 2.76 & 0.11 & 2.88 & -0.24 \\
\hline $6 \mathrm{H}-4,80-82$ & 48.10 & 2.82 & 0.08 & & \\
\hline $6 \mathrm{H}-5,80-82$ & 49.60 & 2.83 & 0.09 & & \\
\hline $6 \mathrm{H}-6,80-82$ & 51.10 & 2.75 & -0.08 & 2.82 & -0.09 \\
\hline $7 \mathrm{H}-1,80-82$ & 53.30 & 2.80 & 0.35 & & \\
\hline $7 \mathrm{H}-2,80-82$ & 54.80 & 3.37 & 0.78 & & \\
\hline $7 \mathrm{H}-4,80-82$ & 57.80 & 2.22 & 1.26 & & \\
\hline $8 \mathrm{H}-1,80-82$ & 63.00 & 2.57 & 0.66 & 2.53 & 0.22 \\
\hline $8 \mathrm{H}-2,80-82$ & 64.50 & 2.71 & 0.78 & & \\
\hline $9 \mathrm{H}-1,80-82$ & 72.60 & 2.48 & 0.66 & & \\
\hline $9 \mathrm{H}-3,80-82$ & 75.60 & 2.67 & 0.41 & & \\
\hline $9 \mathrm{H}-4,80-82$ & 77.10 & & 2.74 & 0.37 & \\
\hline $9 \mathrm{H}-5,80-82$ & 78.60 & 2.47 & 0.85 & 1.80 & 0.46 \\
\hline $9 \mathrm{H}-6,80-82$ & 80.10 & 2.70 & 0.15 & & \\
\hline $10 \mathrm{H}-1,80-82$ & 82.30 & 2.49 & 0.62 & & \\
\hline $10 \mathrm{H}-2,80-82$ & 83.00 & & 2.23 & 0.84 & \\
\hline $10 \mathrm{H}-4,80-82$ & 86.80 & & 1.55 & 0.74 & \\
\hline $10 \mathrm{H}-5,80-82$ & 88.30 & 1.76 & 1.12 & 1.73 & 0.80 \\
\hline $11 \mathrm{H}-1,80-82$ & 92.00 & 1.71 & 0.51 & 1.72 & 0.30 \\
\hline $11 \mathrm{H}-2,80-82$ & 93.50 & & 2.04 & 0.53 & \\
\hline $11 \mathrm{H}-3,80-82$ & 95.00 & 1.99 & 0.68 & 1.98 & 0.51 \\
\hline $11 \mathrm{H}-4,80-82$ & 96.50 & & 2.04 & 0.48 & \\
\hline $11 \mathrm{H}-5,80-82$ & 98.00 & 1.92 & 1.18 & 1.71 & 0.36 \\
\hline $12 \mathrm{H}-1,80-82$ & 101.60 & & 1.98 & 0.58 & \\
\hline $12 \mathrm{H}-2,80-82$ & 103.10 & 1.77 & 1.05 & 1.87 & 0.83 \\
\hline $12 \mathrm{H}-3,80-82$ & 104.60 & 1.83 & 0.95 & 1.91 & 0.83 \\
\hline $12 \mathrm{H}-4,80-82$ & 106.10 & 1.87 & 1.03 & 1.90 & 0.61 \\
\hline $12 \mathrm{H}-5,80-82$ & 107.60 & 1.99 & 0.77 & 1.85 & 0.72 \\
\hline $12 \mathrm{H}-6,80-82$ & 109.10 & & 1.99 & 0.68 & \\
\hline $13 \mathrm{H}-3,80-82$ & 114.30 & & 1.89 & 1.01 & \\
\hline $13 \mathrm{H}-4,80-82$ & 115.80 & & 1.79 & 0.99 & \\
\hline $13 \mathrm{H}-5,80-82$ & 117.30 & & 2.03 & 1.27 & \\
\hline $13 \mathrm{H}-6,80-82$ & 118.80 & & 1.76 & 1.39 & \\
\hline $14 \mathrm{H}-1,80-82$ & 120.90 & & 1.59 & 1.06 & \\
\hline $14 \mathrm{H}-2,80-82$ & 122.40 & 1.13 & 0.88 & & \\
\hline $14 \mathrm{H}-3,80-82$ & 123.90 & 1.15 & 1.04 & 1.18 & 0.94 \\
\hline $14 \mathrm{H}-4,80-82$ & 125.40 & & 1.40 & 1.05 & \\
\hline $14 \mathrm{H}-5,80-82$ & 126.90 & & 1.30 & 1.19 & \\
\hline $14 \mathrm{H}-6,80-82$ & 128.40 & & 1.29 & 1.28 & \\
\hline $15 \mathrm{H}-1,80-82$ & 130.60 & & 1.15 & 1.11 & \\
\hline $15 \mathrm{H}-3,80-82$ & 133.60 & & 1.23 & 0.61 & \\
\hline $15 \mathrm{H}-4,80-82$ & 135.10 & & 1.14 & 0.69 & \\
\hline $15 \mathrm{H}-6,80-82$ & 138.10 & & 1.22 & 1.05 & \\
\hline $16 \mathrm{H}-2,80-82$ & 141.80 & & 1.23 & 1.04 & \\
\hline $16 \mathrm{H}-6,19-21$ & 147.19 & & 0.96 & 1.19 & \\
\hline $18 \mathrm{H}-4,80-82$ & 161.10 & & 0.70 & 0.96 & \\
\hline
\end{tabular}

Note: Analyses were conducted on benthic foraminifers Gyroidinoides sp. and Uvigerina sp. 
752A Oxygen isotopes

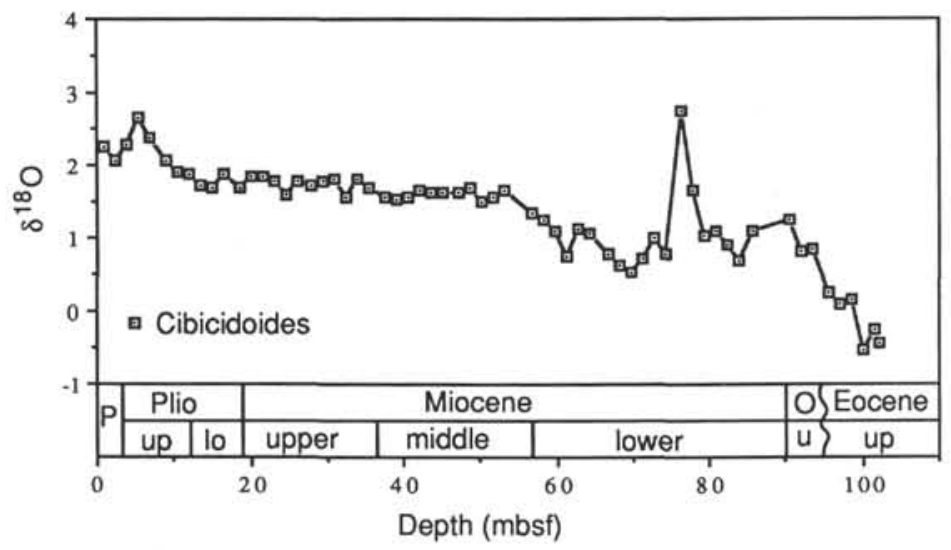

752A Carbon isotopes

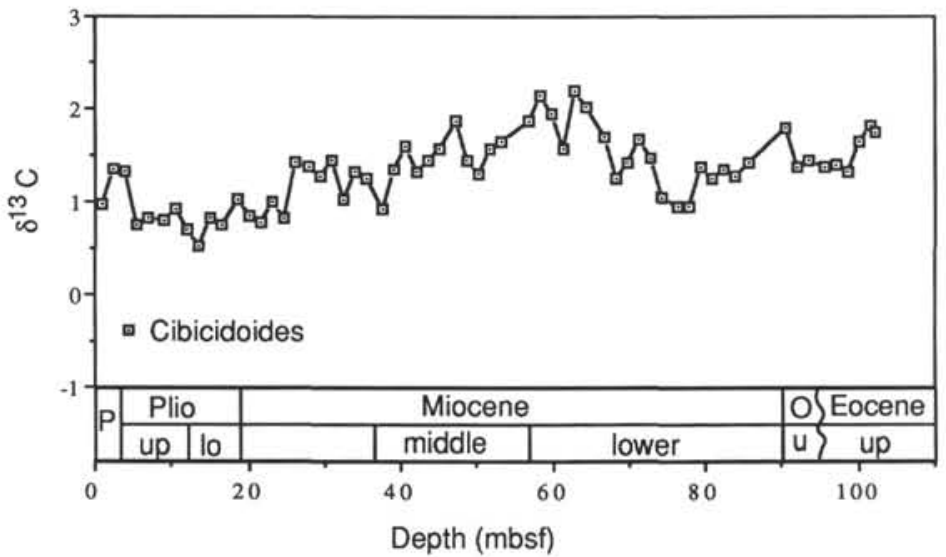

Figure 2. Oxygen and carbon isotopic record of Cenozoic oozes from Hole 752A. 
754A Oxygen isotopes

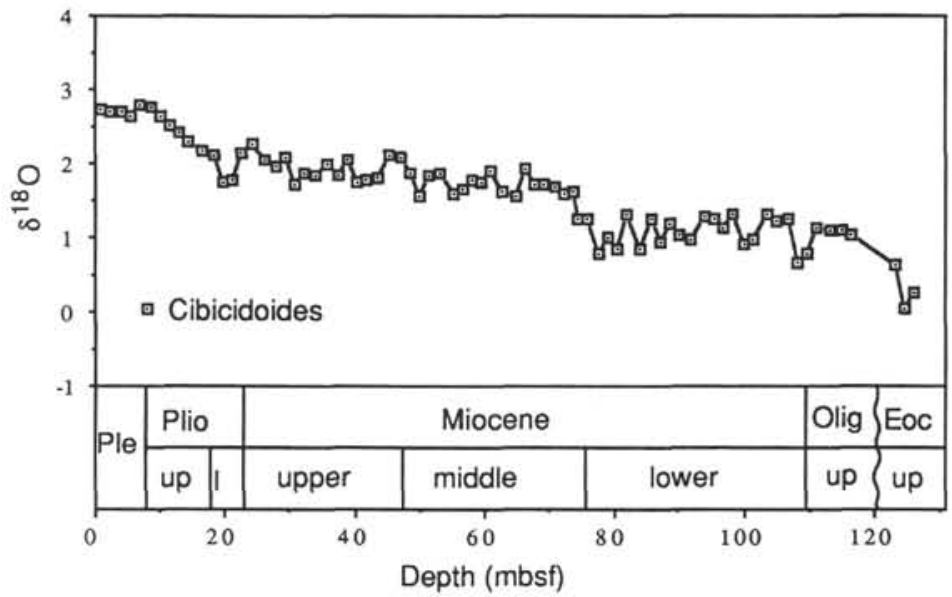

754A Carbon isotopes

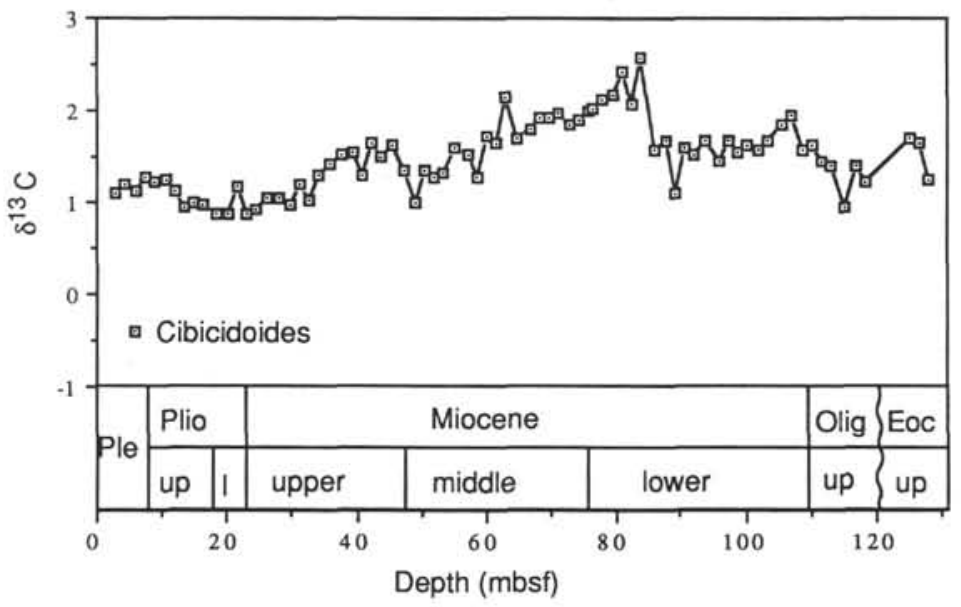

Figure 3. Oxygen and carbon isotopic record of Cenozoic oozes from Hole 754A. 
756B Oxygen isotopes

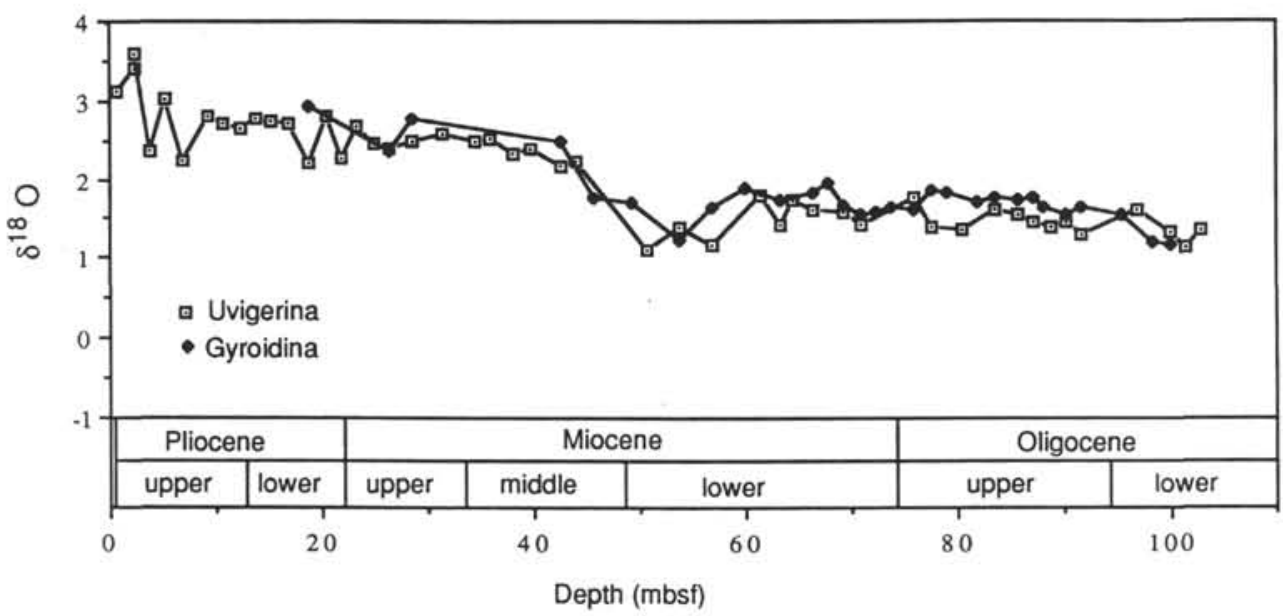

756B Carbon isotopes

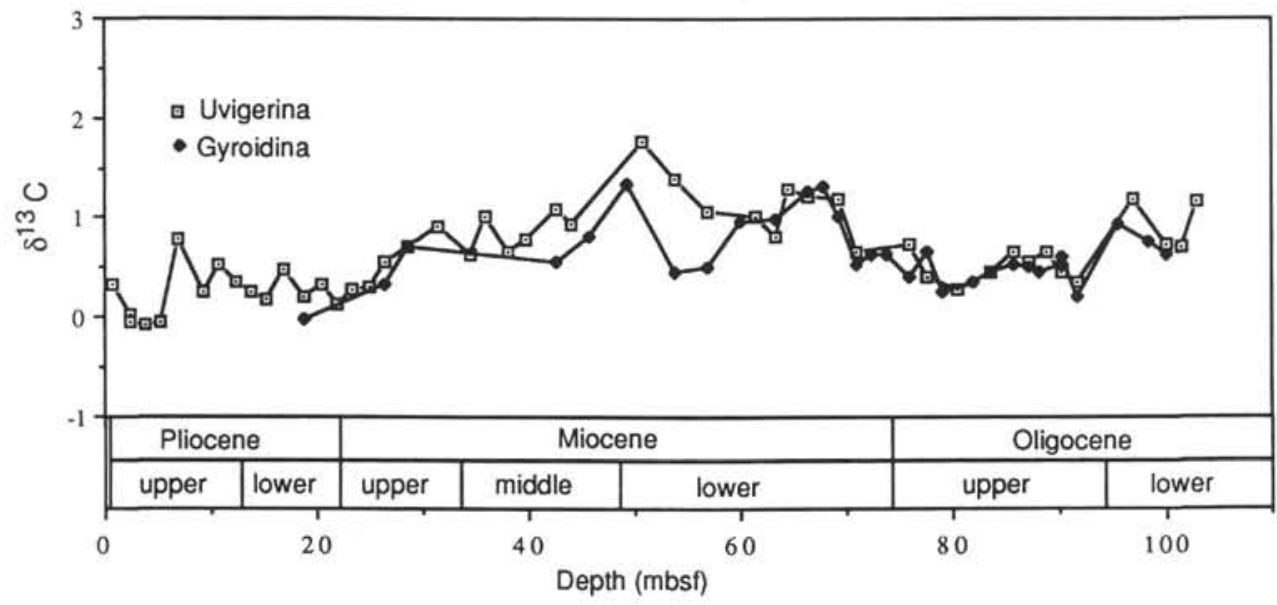

Figure 4. Oxygen and carbon isotopic record of Cenozoic oozes from Hole 756B.
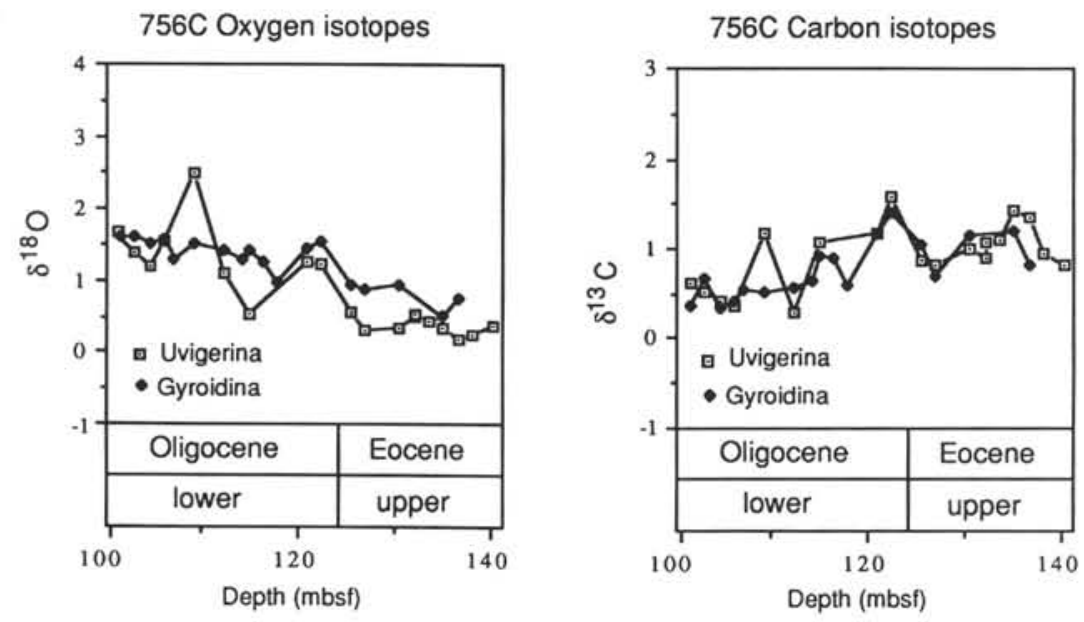

Figure 5. Oxygen and carbon isotopic record of Cenozoic oozes from Hole 756C. 
757B Oxygen isotopes

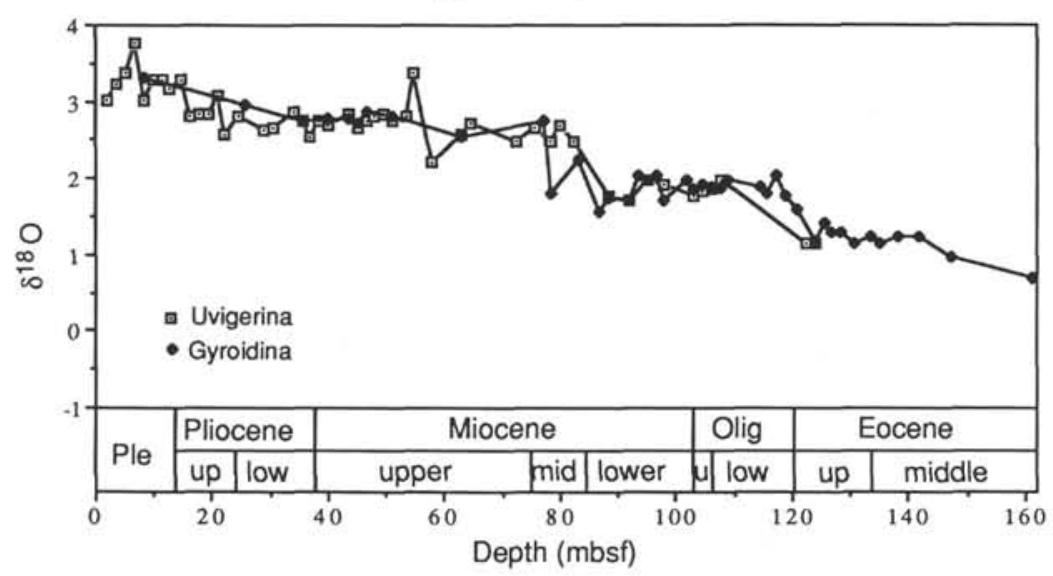

757B Carbon isotopes

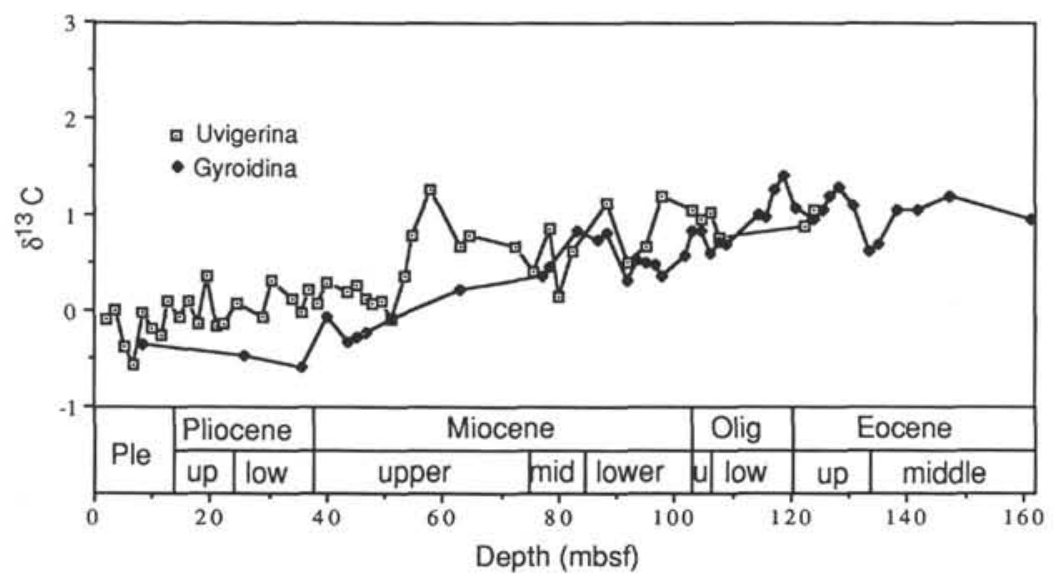

Figure 6. Oxygen and carbon isotopic record of Cenozoic oozes from Hole 757B. 